\title{
Using dynamic pupillometry as a simple screening tool to detect autonomic neuropathy in patients with diabetes: a pilot study
}

\author{
Giselle L Ferrari1,2, Jefferson LB Marques3,4, Rajiv A Gandhi ${ }^{4}$, Simon R Heller ${ }^{4}$, Fábio K Schneider2, Solomon Tesfaye4 \\ and Humberto R Gamba*2
}

\section{* Correspondence: \\ humberto@utfpr.edu.br \\ 2 School of Electrical Engineering and Applied Computer Sciences-CPGEI, Federal University of \\ Technology-Paraná, Curitiba, PR - Brazil \\ Full list of author information is}

\begin{abstract}
Background: Autonomic neuropathy is a common and serious complication of diabetes. Early detection is essential to enable appropriate interventional therapy and management. Dynamic pupillometry has been proposed as a simpler and more sensitive tool to detect subclinical autonomic dysfunction. The aim of this study was to investigate pupil responsiveness in diabetic subjects with and without cardiovascular autonomic neuropathy (CAN) using dynamic pupillometry in two sets of experiments.
\end{abstract}

Methods: During the first experiment, one flash was administered and the pupil response was recorded for $3 \mathrm{~s}$. In the second experiment, 25 flashes at 1-s interval were administered and the pupil response was recorded for $30 \mathrm{~s}$. Several time and pupil-iris radius-related parameters were computed from the acquired data. A total of 24 diabetic subjects (16 without and 8 with (AN) and 16 healthy volunteers took part in the study.

Results: Our results show that diabetic subjects with and without CAN have sympathetic and parasympathetic dysfunction, evidenced by diminished amplitude reflexes and significant smaller pupil radius. It suggests that pupillary autonomic dysfunction occurs before a more generalized involvement of the autonomic nervous system, and this could be used to detect early autonomic dysfunction.

Conclusions: Dynamic pupillometry provides a simple, inexpensive, and noninvasive tool to screen high-risk diabetic patients for diabetic autonomic neuropathy.

\section{Background}

Diabetic autonomic neuropathy (DAN) is the least recognized and understood complications of diabetes, despite its significant negative impact on survival and quality of life in people with diabetes [1]. It can involve the entire autonomic nervous system. Among the most serious DAN is cardiovascular autonomic neuropathy (CAN), which encompasses damage to the autonomic nerve fibers that innervate the heart, affecting heart rate and blood pressure control and vascular dynamics. Diabetes-related CAN occur in one-quarter of the subjects with Type 1 diabetes and in one-third of those with Type 2 diabetes. CAN is associated with increased mortality, silent myocardial ischemia, and may even predict the development of stroke [2]. While DAN can be clinically evident, manifested by dysfunction of one or more organ systems (e.g. cardiovascular, gastrointestinal, genitourinary, sudomotor, or ocular) [3], it is frequently subclinical and asymptomatic. 
A recent statement of the ADA [4] concerning diabetic neuropathies recommends that screening should be instituted at diagnosis of Type 2 diabetes and 5 years after the diagnosis of Type 1 diabetes. Screening might comprise a history and an examination for the signs of autonomic dysfunction. Earlier identification of these high-risk individuals is clearly important, as it would allow for the deployment of management strategies to improve long-term outcomes. If diagnosis is delayed until the development of symptoms, the outcome is extremely poor, with a 5 -year survival rate of only $50 \%$ [5].

Although several conventional autonomic function tests exist to aid clinicians in the evaluation of DAN, they require specific equipments and well-trained personnel, and are time-consuming to perform [6]. Additionally, these tests typically require active patient participation and compliance, leading to their use in a minority of subjects, mainly those with advanced autonomic neuropathy who have already developed symptoms. Therefore, the development of new approaches for the early identification of DAN, i.e., before the onset of symptoms, is desired. This would allow for interventions and management that may result in reduced long-term complications, as previously reported [7]. In this context, simple and inexpensive approach to screen large number of subjects with diabetes for autonomic neuropathy would be valuable to minimize the enormous social and economic burden caused by DAN, especially for subjects at a much higher risk, such as those with undiagnosed CAN. The pupil dynamics analysis, i.e., dynamic pupillometry, has the potential for supporting such a screening approach.

Pupil size, shape, and reactivity to light have been used as indicators of neurological function in brain-injured patients, particularly in comatose patients. There are circular and radial muscles that control the size of the pupil. The former is innervated by parasympathetic fibers, and the latter, by sympathetic fibers. Thus, the pupil radius is controlled by both the sympathetic and parasympathetic autonomic nervous system in response to environmental light, a mechanism called the pupil light reflex. Therefore, the pupillary radius response to an external light stimulus might provide an indirect means to assess the integrity of neuronal pathways controlling pupil size [8] and an early indication of DAN [9].

Sympathetic stimulation of $\alpha-1$ adrenergic receptors causes the contraction of the radial muscle, and subsequent dilatation of the pupil (mydriasis). Conversely, parasympathetic stimulation causes contraction of the circular muscle and constriction of the pupil (miosis). The pupillary reflex is mediated by acetylcholine and noradrenaline, causing miosis and mydriasis, respectively. Thus, change in pupil size in response to a light stimulus is based on a functional equilibrium between sympathetic and parasympathetic activity [10].

It is therefore reasonable to expect that generalized conditions of the autonomic system will affect the pupil response to the light stimulus [11]. Parasympathetic dysfunction might cause relative mydriasis of the pupil in light conditions and diminished constrictor reflexes with or without pupillotonia, which is thought to result from aberrant re-innervations. Sympathetic dysfunction might cause relative miosis of the pupil in the dark, increased re-dilatation lag, and attenuation of the startle reflex, as observed in Horner's syndrome [12]. Investigations in subjects with diabetes have revealed abnormal responses of the pupil-radius modulation in some subjects, especially in those with diabetic neuropathy [9,13-18], supporting the potential of pupillometry to be used as a screening tool for autonomic neuropathy in the diabetic population [15-17]. 
Previous studies have described the resting pupil radius as a sympathetic parameter, and the reflex amplitude as a parasympathetic parameter in healthy and diabetic subjects [9,13-18]. However, there is very little evidence with regard to the relationship between the pupil-iris ratio and other time-related parameters with autonomic neuropathy in diabetes subjects with and without CAN. In this study, the results of an investigative study of the pupil responsiveness to light flashes in healthy and diabetic subjects with and without clinical evidence of CAN has been presented. The study was performed with a custom-built dynamic pupillometer [19] that allows the computation of a larger number of parameters when compared with commercially available pupillometers.

\section{Methods}

\section{Subjects}

This study comprised three groups: Group $1-16$ healthy volunteers ( 9 males and 7 females); Group 2 - 16 diabetic subjects without CAN (15 males and 1 females); and Group 3 - 8 diabetic subjects with CAN (7 males and 1 females). The study was conducted in the Diabetes Centre, Royal Hallamshire Hospital, University of Sheffield, UK.

Informed consent was obtained from all participants. Subjects were not receiving any drugs affecting sympathetic or parasympathetic pupillary function, and those with past history of ocular operations, nonsymmetrical pupil, misshapen pupil, or conditions affecting pupillary reflexes were excluded [20]. All diabetic subjects underwent standard blood and biochemical laboratory tests.

Owing to the experiment setup, only the right side of each participant was accessible for pupillometry. The pupil reaction was assessed twice: first with one flash, and $5 \mathrm{~min}$ later with 25 flashes. Both tests were performed in the right eye, and all the subjects were tested between 9 and $12 \mathrm{am}$. For all patients, at least $8 \mathrm{~h}$ of sleep was required in the preceding night without any hypoglycemic episode.

The pupil stimulator and response recorder

Figure 1 illustrates the setup of the instrument, named Pupil Stimulator and Response Recorder (PSRR), developed to stimulate and record the pupil response to flashes of white light [19]. It consisted of a commercial monochromatic CCD analog video camera (EIA-RS-170 standard) that captures an image frame every 1/30 seconds; four infrared (IR) light emitter diodes (LEDs) with a diameter of $5 \mathrm{~mm}$; five high-intensity bright white

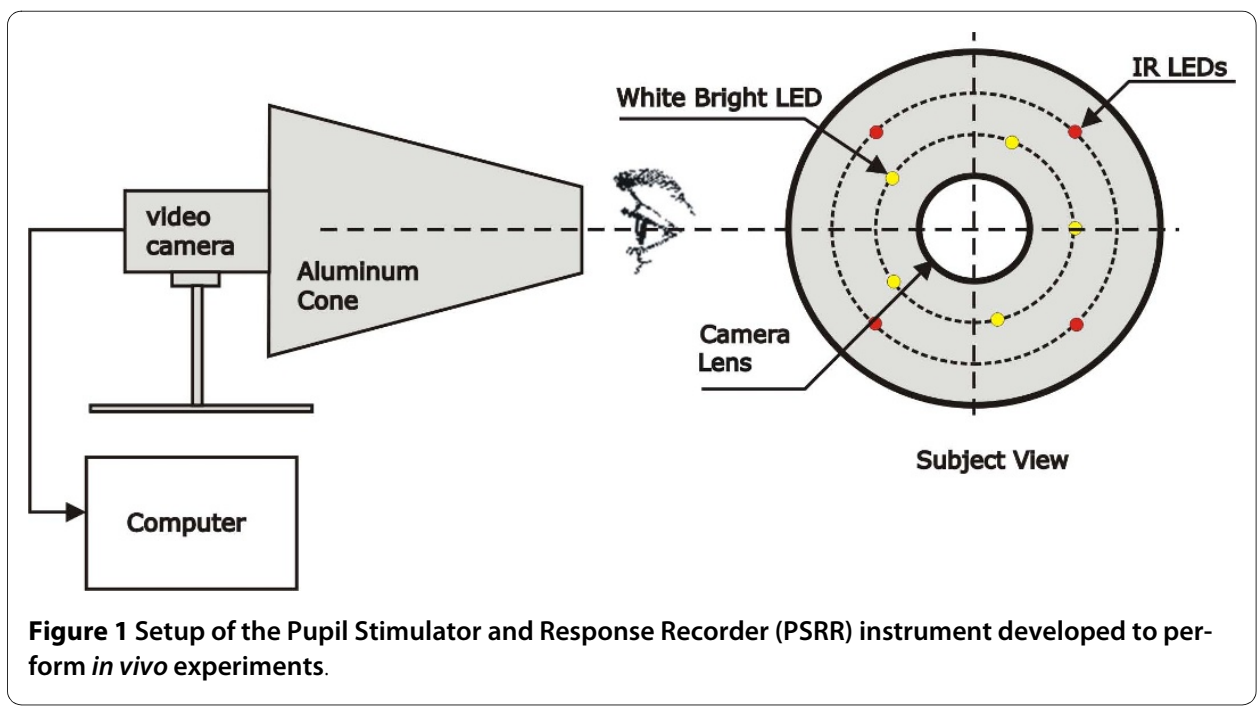


LEDs with a diameter of $8 \mathrm{~mm}$; a 17-cm height aluminum cone section with extremity diameters of 14.5 and $5.6 \mathrm{~cm}$; an electronic current supply to the LEDs; a built unit for timing control based on the microcontroller PIC16F873 (Microchip Technology Inc., Arizona, USA); and a Pentium IV $3 \mathrm{GHz}$ Notebook with a PCMCIA frame grabber to record and process the pupil images. The aluminum cone was fixed in a printed circuit board (that hold the IR and white LEDs), which was then fixed in the CCD camera. The IR and the high-intensity bright white LEDs were symmetrically placed around the CCD lens, as illustrated in Figure 1. The IR LEDs promoted the illumination with a high pupil to iris contrast, but without producing any pupil response. The lens focus was manually adjusted by rotating the aluminum cone. The pupil and the aluminum cone wall acted as a mirror, reflecting the IR LEDs light. The light reflection in the cone wall caused too many artifacts in the pupil image, making the pupil-iris edge detection difficult during the image processing phase. To avoid these artifacts, the aluminum cone interior was blackened.

The PSRR can stimulate the pupil with one flash or a series of 25 flashes $(1-\mathrm{Hz}$ frequency), with fixed intensity $(250 \mathrm{~cd})$ and duration $(10 \mathrm{~ms})$. The changes in the radius of the pupil were evaluated as a function of several time-dependent and pupil-iris radius ratio parameters.

The pupil radius was automatically determined for each frame in the recorded video stream. The offline image processing algorithm was implemented using MATLAB (The MathWorks Inc., Massachusetts, USA) environment and was divided into three major steps: elimination of the IR LEDs light reflex within the pupil; enhancement of the image borders; and pupil and iris edge detection.

Figure 2 shows the images obtained in some of the major algorithms steps until the best circle that fits the pupil is found. Figure 2(a) shows the region of interest (ROI) that is cropped from the original image. First, the IR LED light reflection was detected using the Canny edge detector with a threshold set to 0.8 . The detector returned one pixel line that makes a frontier between the white reflected and the dark pupil regions. These contours were then filled, forming a reference mask to change the white pixel in the pupil by a gray value of 35 . It is important to note that owing to our image acquisition system capturing the eye images under the same illumination condition, the pupil darkness of every subject has a gray value around 35 . Figure 2(b) and 2(c) shows the mask and the eye image with the IR LED light reflection removed. This image was then edge enhanced using a high-boost filtering [21], followed by an intensity transformation through a 0.6gamma function. The result is shown in Figure 2(d). A Canny edge detector with a 0.5threshold was then applied to find the frontier pixels between the pupil and iris, as shown in Figure 2(e). To determine the pupil radius, it was found that the best solution was by convolving the image shown in Figure 2(e) with contour circle masks with different radius (20-90 pixels, with 0.2 pixels of resolution), and determining the circle radius that gives the maximum correlation.

This algorithm was even able to determine the pupil radius in those cases where the pupil was under the eyelid or eyelashes, as shown in Figure 3(a) and 3(b).

The iris radius was automatically determined for each frame in the recorded video stream using the Hough Transform, followed by a canny edge detector algorithm [21]. 


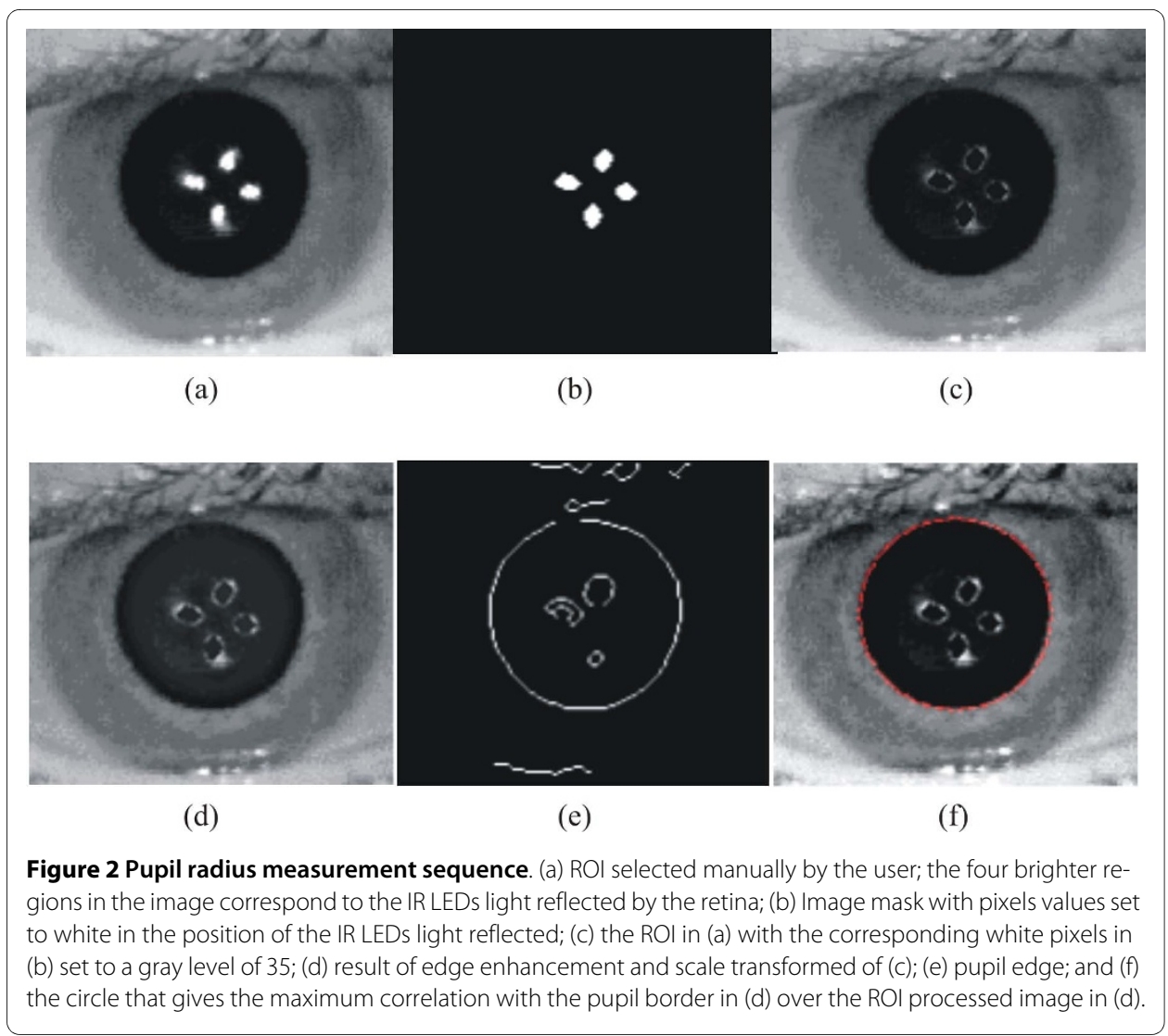

\section{Experiments}

All the subjects underwent two experiments. In the first experiment, the subject darkadapted for 2 min leaning on the cone before a single light flash was administered. The pupil response was recorded for $3 \mathrm{~s}$. During this period, the subject was instructed to avoid or minimize blinking.

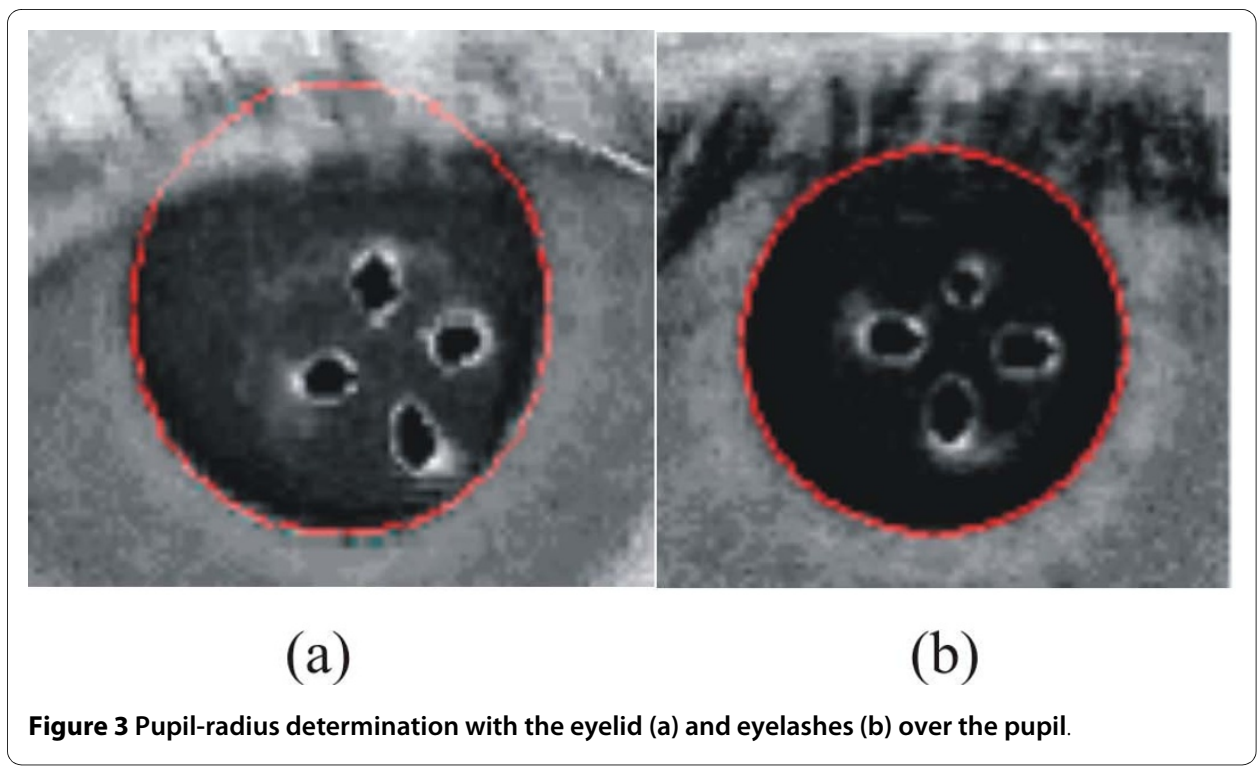


In the second experiment, the subject dark-adapted for 2 min before 25 flashes were administered at 1-s interval. The pupil response was recorded for $30 \mathrm{~s}$. In both the experiments, the other eye was covered with a black fabric to avoid any external light interference.

\section{Single light flash parameters}

Figure 4 shows a typical pupil response obtained from a healthy volunteer. It shows the radius of the pupil in each captured frame in pixels as a function of time.

The pupil reflex evaluation parameters can be defined as follows [10]:

- Pupil-iris radius ratio in the frame preceding the flash, \#7 in Figure 4, divided by iris radius;

- Latency from flash exposure to the start of constriction (when pupil radius decreases to $90 \%$ of pre-flash value), \#2 in Figure 4;

- Pupil-iris radius ratio (\#6 in Figure 4 divided by iris radius) and latency (\#4 in Figure 4) to the smallest size of the pupil;

- During the recovery phase: pupil-iris radius ratio (\#5 in Figure 4 divided by iris radius) and latency to plateau at $75 \%$ of pre-flash pupil radius (\#1 in Figure 4);

- Duration of constriction (\#3 in Figure 4);

- Reflex amplitude - resting pupil radius (\#7 in Figure 4) minus minimum pupil radius after the light stimulus (\#6 in Figure 4);

- Velocity of constriction - changing rate in the pupil radius over the reflex amplitude time interval: reflex amplitude divided by \#3 in Figure 4.

\section{Twenty-five light flashes parameters}

Figure 5 shows a typical pupil response obtained from a healthy volunteer. It shows the radius of the pupil in pixels for each captured frame as a function of time and the main parameters measured.

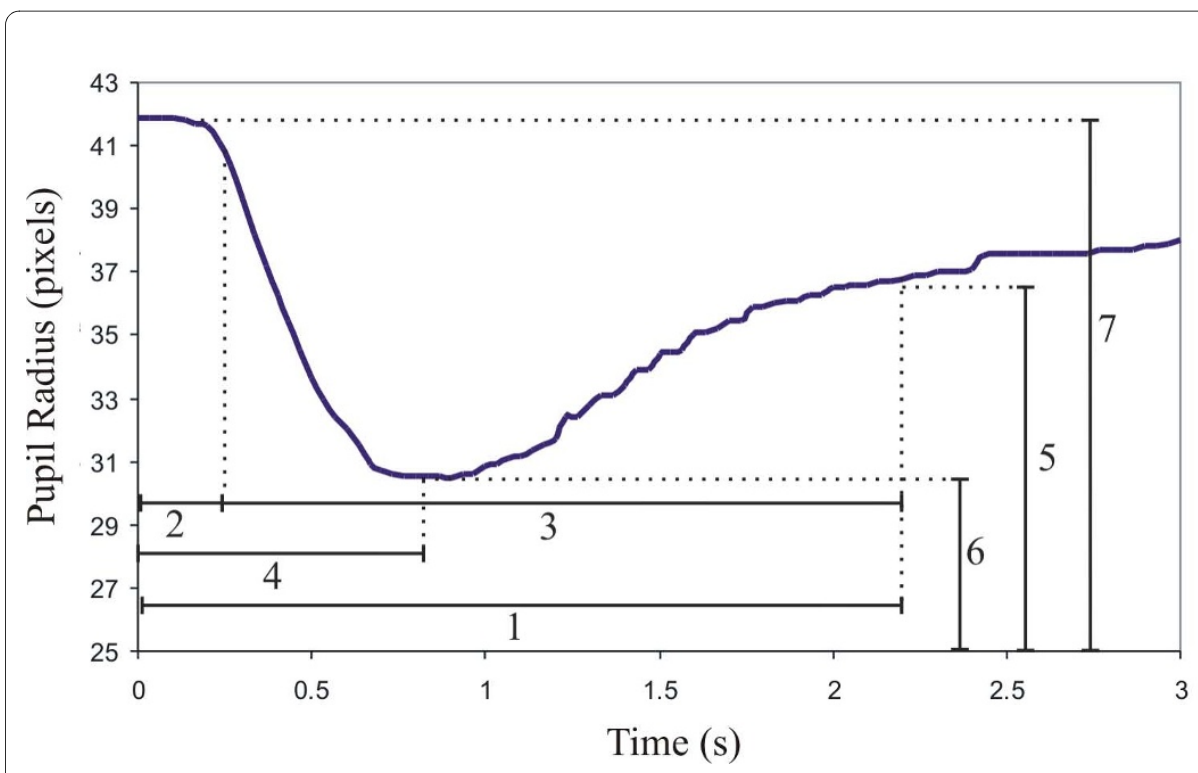

Figure 4 Typical pupil reflex of a healthy volunteer after a 10-ms light flash intensity of $250 \mathrm{~cd}$ was triggered at zero second. The indicated parameters are defined as follows: 1-Latency time to reach the plateau at $75 \%$ of pre-flash pupil radius; 2-Latency time to the beginning of constriction; 3-Duration of constriction; 4Latency to the largest constriction; 5- Radius of pupil on reaching the plateau; 6-Radius of pupil at largest constriction; 7-Radius of pupil before flash. 


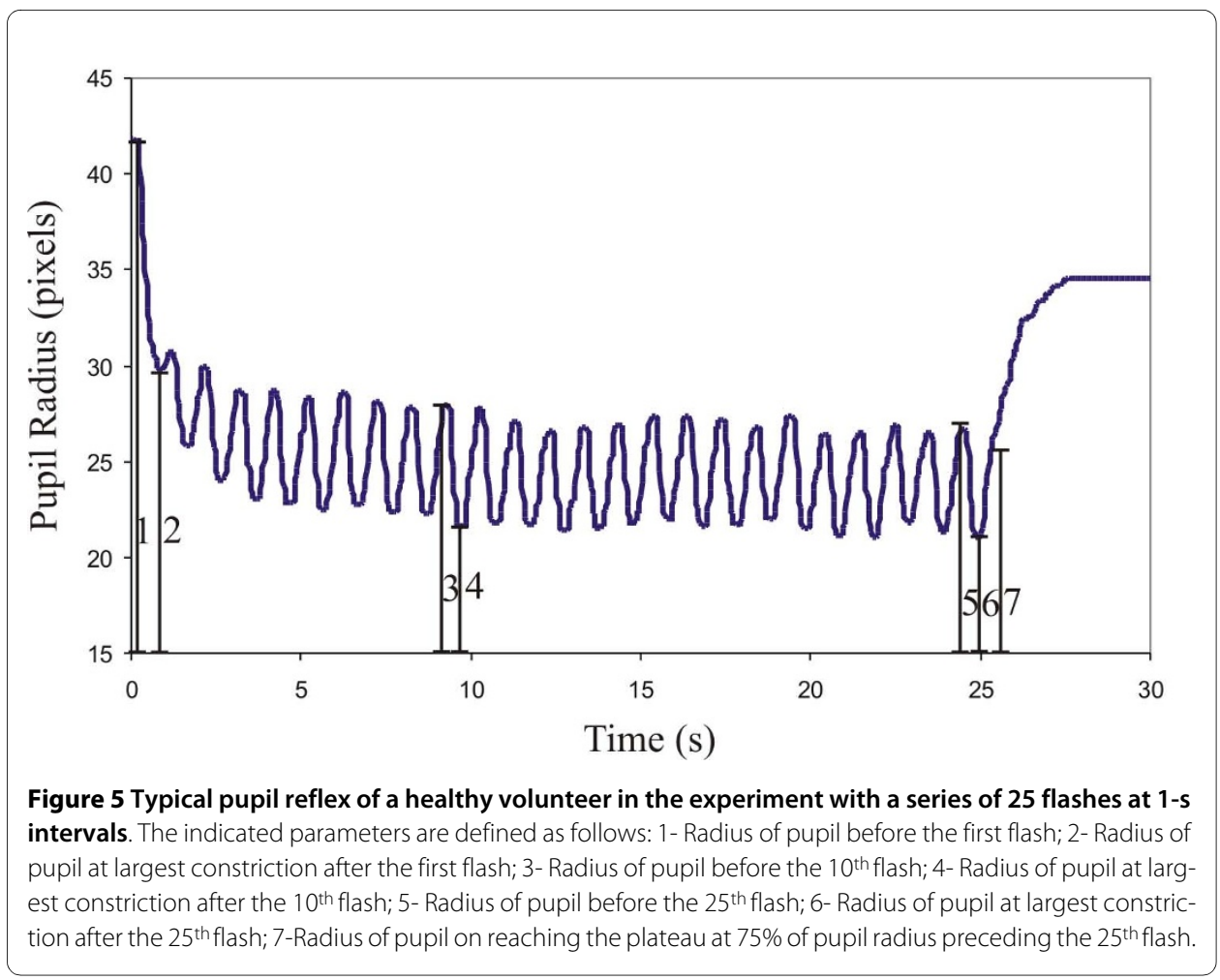

For the 25-flashes experiment, the pupil reflex evaluation parameters can be defined as follows [10]:

- Pupil-iris ratio in the frame preceding the $10^{\text {th }}$ (\#3 in Figure 5 divided by iris radius) and $25^{\text {th }}$ flash (\#5 in Figure 5 divided by iris radius);

- Pupil-iris ratio of the smallest size of the pupil after the $10^{\text {th }}$ (\#4 in Figure 5 divided by iris radius) and $25^{\text {th }}$ flash ( $\# 6$ in Figure 5 divided by iris radius);

- Latency from flash exposure to the start of constriction after the $10^{\text {th }}$ and $25^{\text {th }}$ flash;

- Latency to the smallest size of the pupil after the $10^{\text {th }}$ and $25^{\text {th }}$ flash;

- During the recovery phase: pupil-iris ratio (\#7 in Figure 5 divided by iris radius) and latency to plateau when the pupil radius reaches $75 \%$ of the pre-flash value;

- Duration of constriction.

\section{Assessment for CAN}

A standardized set of cardiovascular tests was used to assess the prevalence of CAN. The tests were performed after a resting period of $10 \mathrm{~min}$ to ensure that the heart rate was at its baseline values. The tests included recording the electrocardiogram (ECG) to determine the heart rate variability (HRV), and measurements of systolic and diastolic blood pressure (BP). Four tests of HRV and one test of BP control were performed and evaluated according to standard criteria [22]. Recordings were carried out at rest (HRV and BP), deep breathing (HRV), Valsalva maneuver (HRV), and active standing up (HRV and $\mathrm{BP})$. The total duration of these tests was approximately $30 \mathrm{~min}$. Diabetic subjects were classified as having CAN if the results of two or more of these five autonomic function tests were below age-adjusted normative values [22].

Statistical analysis

Statistical analysis for all the variables included the calculation of mean and standard deviation (SD). One-way ANOVA and post-hoc Bonferroni statistical tests were used to 
compare the pupillography results between the groups. Comparisons involving the diabetes group (without CAN vs. with CAN) were carried out using Kruskal-Wallis Test. SPSS version 15.0 (SPSS Inc., Chicago, USA) was used to analyze the data, and $p$ values less than 0.05 were considered statistically significant.

\section{Results}

\section{Single light flash experiment}

The mean and SD values for each parameter used to evaluate the response of the pupil reaction to a single flash and the statistical significance tests are shown in Table 1.

There was no significant difference between the three groups with regard to age ( $p>$ $0.05)$. The duration of diabetes did not differ in the diabetic subjects with $(22 \pm 10$ years

Table 1: Subjects' baseline characteristics and pupillometry parameters

\begin{tabular}{|c|c|c|c|c|c|c|}
\hline \multirow[t]{2}{*}{ Parameters } & \multirow[t]{2}{*}{ Healthy Volunteers } & \multicolumn{2}{|c|}{ Diabetes } & \multicolumn{3}{|c|}{$p$ values } \\
\hline & & Without CAN & With CAN & $p_{1}$ & $p_{2}$ & $p_{3}$ \\
\hline Number of subjects & 16 & $\begin{array}{c}16 \\
\text { (9 Type 1, } 7 \text { Type 2) }\end{array}$ & $\begin{array}{c}8 \\
\text { (3 Type 1, } 5 \text { Type 2) }\end{array}$ & - & - & - \\
\hline Gender (M/F) & $9 / 7$ & $15 / 1$ & $7 / 1$ & - & - & - \\
\hline Age (years) & $44 \pm 13$ & $50 \pm 11$ & $51 \pm 5$ & ns & ns & ns \\
\hline $\begin{array}{l}\text { Duration of diabetes } \\
\text { (years) }\end{array}$ & - & $20 \pm 10$ & $22 \pm 10$ & - & - & ns \\
\hline $\mathrm{HbA} 1 \mathrm{c}(\%)$ & - & $8.3 \pm 1.1$ & $9.4 \pm 2.2$ & - & - & ns \\
\hline $\begin{array}{l}\text { Systolic blood pressure } \\
(\mathrm{mmHg})\end{array}$ & $120 \pm 13$ & $141 \pm 20$ & $147 \pm 16$ & * & * & ns \\
\hline $\begin{array}{l}\text { Diastolic blood pressure } \\
(\mathrm{mmHg})\end{array}$ & $74 \pm 6$ & $77 \pm 9$ & $87 \pm 10$ & ns & * & ns \\
\hline Ratio $\mathrm{P} / \mathrm{l}$ in darkness & $0.55 \pm 0.056$ & $0.42 \pm 0.045$ & $0.35 \pm 0.077$ & $\$$ & $\$$ & * \\
\hline Latency to constriction (s) & $0.20 \pm 0.078$ & $0.26 \pm 0.070$ & $0.29 \pm 0.100$ & ns & * & ns \\
\hline $\begin{array}{l}\text { Ratio } \mathrm{P} / \mathrm{I} \text { at largest } \\
\text { constriction }\end{array}$ & $0.40 \pm 0.050$ & $0.30 \pm 0.031$ & $0.26 \pm 0.065$ & $\$$ & $\$$ & ns \\
\hline $\begin{array}{l}\text { Latency to largest } \\
\text { constriction (s) }\end{array}$ & $0.90 \pm 0.147$ & $1.01 \pm 0.192$ & $0.91 \pm 0.089$ & ns & ns & ns \\
\hline Reflex amplitude (pixels) & $9.62 \pm 1.99$ & $7.40 \pm 1.90$ & $5.35 \pm 2.14$ & \# & \# & ns \\
\hline Ratio $\mathrm{P} / \mathrm{l}$ of plateau & $0.50 \pm 0.055$ & $0.37 \pm 0.046$ & $0.32 \pm 0.068$ & $\$$ & $\$$ & ns \\
\hline Latency to plateau (s) & $1.88 \pm 0.539$ & $1.95 \pm 0.744$ & $1.49 \pm 0.331$ & ns & ns & ns \\
\hline Duration of constriction (s) & $1.63 \pm 0.559$ & $1.69 \pm 0.75$ & $1.19 \pm 0.318$ & ns & ns & ns \\
\hline $\begin{array}{l}\text { Velocity of constriction } \\
\text { (pixels/s) }\end{array}$ & $14.57 \pm 5.32$ & $10.28 \pm 3.49$ & $7.87 \pm 3.48$ & $*$ & $\#$ & ns \\
\hline
\end{tabular}

[mean \pm standard deviation] for the single flash experiment. $p_{1}$ - Comparison between healthy volunteers and diabetic subjects without CAN. $p_{2}$-Comparison between healthy volunteers and diabetic subjects with CAN. $p_{3}$ - Comparison between diabetic subjects without and with CAN. ns - not significant, ${ }^{*}-p<0.05$, \# $p<0.01$, and $\$-p<0.001$. 
[mean $\pm \mathrm{SD}])$ and without CAN (20 \pm 10 years), and HbA1c (\%) was not significantly different between these two groups $(9.4 \pm 2.2$ and $8.3 \pm 1.1$, respectively).

Systolic blood pressure was significantly lower $(p<0.05)$ in healthy volunteers $(120 \pm$ $13 \mathrm{mmHg})$ when compared with diabetic subjects without CAN (141 $\pm 20 \mathrm{mmHg})$ and diabetic subjects with CAN (147 $\pm 16 \mathrm{mmHg})$. Moreover, diastolic blood pressure was significantly greater $(p<0.05)$ in diabetic subjects with CAN $(87 \pm 10 \mathrm{mmHg})$ than in diabetic subjects without CAN (77 $\pm 9 \mathrm{mmHg})$.

Table 1 indicates that the pupil-iris ratio in the frame preceding the light flash was significantly greater $(p<0.001)$ in healthy volunteers $(0.55 \pm 0.056$ [mean+SD]) than in diabetic subjects without CAN $(0.42 \pm 0.045)$ and diabetic subjects with CAN (0.35 \pm 0.077). The remarkable difference in the pupil size in darkness can be seen in Figure 6 which shows the images of a healthy volunteer (a), diabetic subject without CAN (b), and diabetic subject with CAN (c). In Figure 6, the four white spots within the pupil are the reflecting IR LEDs used to illuminate the eye during image recording.

Latency from flash exposure to the start of constriction was significantly longer $(p<$ $0.01)$ in the diabetic subjects with CAN $(0.29 \pm 0.100 \mathrm{~s})$ than in healthy volunteers $(0.20$ $\pm 0.078 \mathrm{~s})$.

Similarly, the pupil-iris ratio in the frame of largest constriction was significantly greater $(p<0.001)$ in healthy volunteers $(0.40 \pm 0.050)$ than in diabetic subjects without CAN $(0.30 \pm 0.031)$ and with CAN $(0.26 \pm 0.065)$.

However, the latency to the largest constriction of the pupil was not significantly different between the groups.

The difference in the pupil size in the frame of largest constriction is illustrated in Figure 7 which shows the images of healthy volunteer (a), diabetic subject without CAN (b), and diabetic subject with CAN (c).

During the recovery phase, pupil-iris ratio in the plateau was significantly greater $(p<$ $0.001)$ in healthy volunteers $(0.50 \pm 0.055)$ than in diabetic subjects with CAN $(0.32 \pm$ $0.068)$ and diabetic subjects without CAN $(0.37 \pm 0.046)$.

No significant differences were found between the groups in the latency to reach the plateau and duration of constriction.

Reflex amplitude measured in pixels was significantly higher $(p<0.01)$ in healthy volunteers $(9.62 \pm 1.99)$ when compared with diabetic subjects without CAN $(7.40 \pm 1.90)$ and diabetic subjects with CAN (5.35 \pm 2.14$)$. Moreover, the velocity of constriction (pixels/s) was significantly higher $(p<0.05)$ in healthy volunteers $(14.57 \pm 5.32)$ when com-

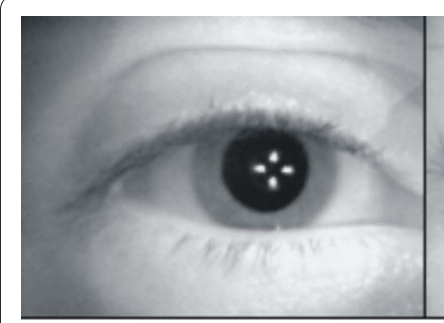

(a)

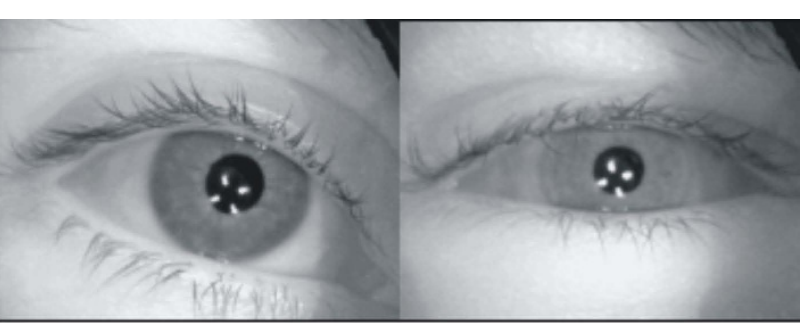

(b) (c)

Figure 6 Recorded IR-illuminated images of the eye of three subjects showing the pupil at its maximum mydriasis for (a) healthy volunteer, (b) diabetic subject without CAN, and (c) diabetic subject with CAN 


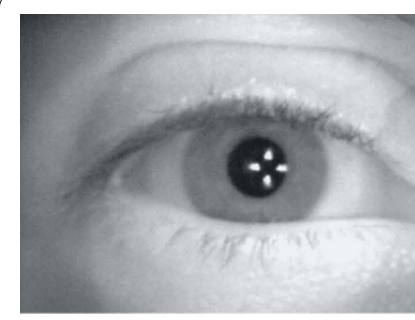

(a)

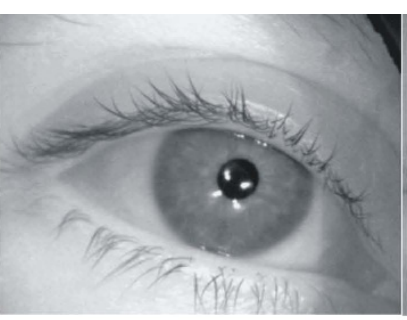

(b)

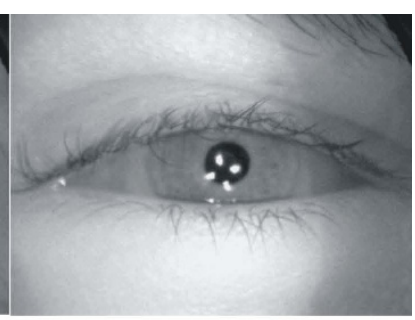

(c)

Figure 7 Recorded IR-illuminated images of the eye of three subjects showing the pupil at its maximum miosis for (a) healthy volunteer, (b) diabetic subject without CAN, and (c) diabetic subject with CAN.

pared with diabetic subjects without CAN $(10.28 \pm 3.49)$ and diabetic subjects with CAN (7.87 \pm 3.48$)$.

No significant difference was found between the diabetic groups, with exception for the $\mathrm{P} / \mathrm{I}$ ratio in darkness.

Figure 8 shows a comparison of the mean and SD of the pupil-iris ratios among the three groups.

Twenty-five light flashes experiment

In the second experiment, one diabetic subject without CAN and three diabetic subjects with CAN blinked more than three times during the experiment and were excluded.

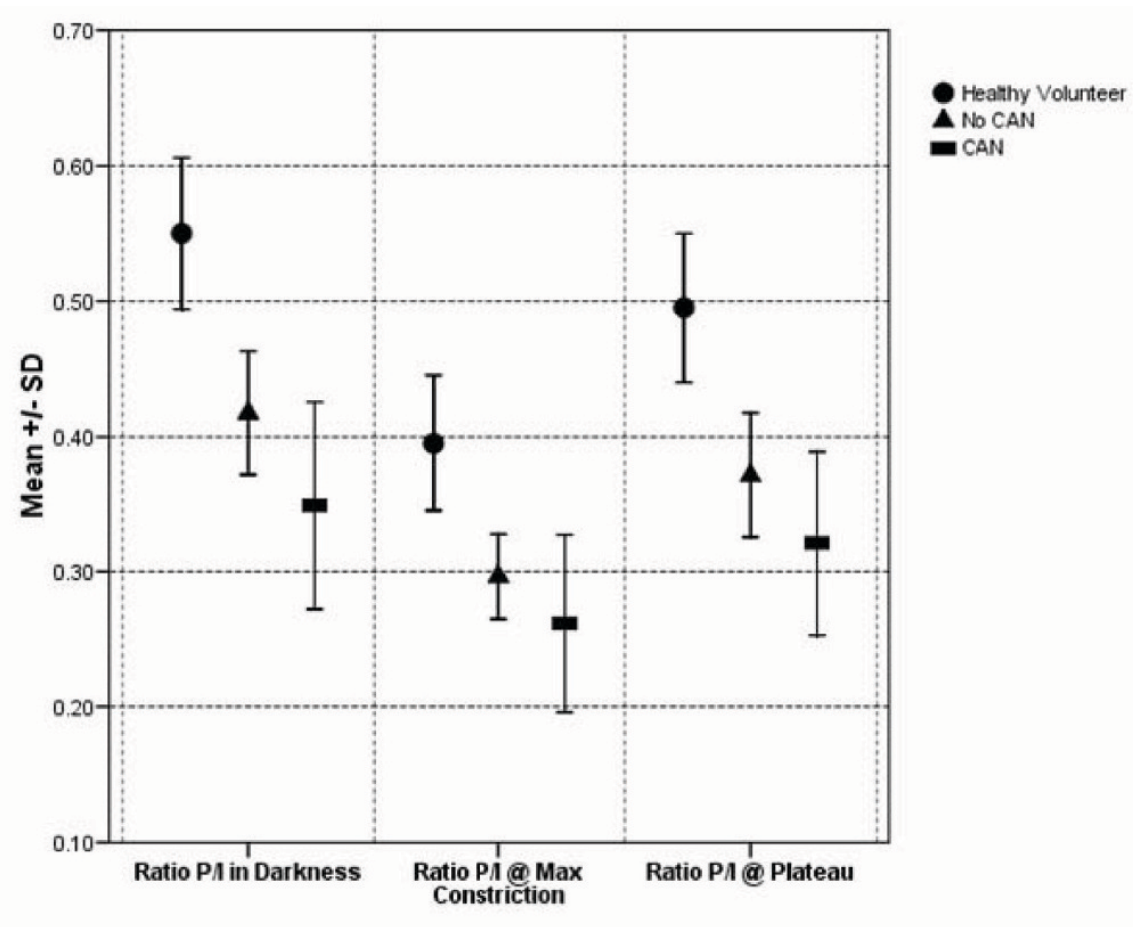

Figure 8 Comparison of mean and standard deviation values of the pupil-iris ratio $(P / I)$ in the largest mydriasis before flash (\#7 in Figure 4), at largest miosis (\#6 in Figure 4), and during the plateau period (\#5 in Figure 4). These values were obtained after one 10-ms light flash in healthy volunteers and diabetic subjects with and without CAN. 
Table 2: Subjects' baseline characteristics and pupillometry parameters

\begin{tabular}{|c|c|c|c|c|c|c|c|}
\hline \multirow[t]{2}{*}{ Flash } & \multirow[t]{2}{*}{ Parameters } & \multirow[t]{2}{*}{ Healthy Volunteers } & \multicolumn{2}{|c|}{ Diabetic } & \multicolumn{3}{|c|}{$p$ values } \\
\hline & & & Without CAN & With CAN & $p_{1}$ & $p_{2}$ & $p_{3}$ \\
\hline & Number of subjects & 16 & $\begin{array}{c}15 \\
\text { (9 Type 1, } 7 \text { Type 2) }\end{array}$ & $\begin{array}{c}5 \\
\text { (2 Type 1, } 3 \text { Type 2) }\end{array}$ & - & - & - \\
\hline & Gender (M/F) & $9 / 7$ & $14 / 1$ & $4 / 1$ & - & - & - \\
\hline & Age (years) & $44 \pm 13$ & $51 \pm 11$ & $49 \pm 4$ & ns & ns & $\mathrm{ns}$ \\
\hline & Duration of diabetes (years) & - & $20 \pm 11$ & $20 \pm 15$ & - & - & ns \\
\hline & HbA1c (\%) & - & $8.3 \pm 1.1$ & $10.2 \pm 2.1$ & - & - & $\mathrm{ns}$ \\
\hline & Systolic blood pressure $(\mathrm{mmHg})$ & $120 \pm 13$ & $143 \pm 20$ & $145 \pm 14$ & * & * & ns \\
\hline & Diastolic blood pressure (mmHg) & $74 \pm 6$ & $78 \pm 9$ & $86 \pm 8$ & ns & * & ns \\
\hline \multirow[t]{4}{*}{$10^{\text {th }}$} & Ratio $\mathrm{P} / \mathrm{I}$ preceding the $10^{\text {th }}$ flash & $0.33 \pm 0.050$ & $0.26 \pm 0.024$ & $0.28 \pm 0.071$ & $\$$ & ns & ns \\
\hline & Latency to constriction (s) & $0.35 \pm 0.139$ & $0.42 \pm 0.126$ & $0.44 \pm 0.192$ & ns & ns & ns \\
\hline & Latency to largest constriction (s) & $0.68 \pm 0.165$ & $0.75 \pm 0.133$ & $0.68 \pm 0.293$ & ns & ns & ns \\
\hline & Ratio $\mathrm{P} / \mathrm{l}$ at largest constriction & $0.28 \pm 0.049$ & $0.24 \pm 0.021$ & $0.27 \pm 0.076$ & * & ns & $\mathrm{ns}$ \\
\hline \multirow[t]{7}{*}{$25^{\text {th }}$} & Ratio $\mathrm{P} / \mathrm{I}$ preceding the $25^{\text {th }}$ flash & $0.32 \pm 0.044$ & $0.26 \pm 0.022$ & $0.30 \pm 0.062$ & $\#$ & ns & $\mathrm{ns}$ \\
\hline & Latency to constriction (s) & $0.31 \pm 0.125$ & $0.36 \pm 0.070$ & $0.40 \pm 0.155$ & ns & ns & ns \\
\hline & Ratio $\mathrm{P} / \mathrm{l}$ at largest constriction & $0.27 \pm 0.046$ & $0.23 \pm 0.020$ & $0.25 \pm 0.063$ & ns & ns & ns \\
\hline & Latency to largest constriction (s) & $0.69 \pm 0.123$ & $0.72 \pm 0.054$ & $0.79 \pm 0.138$ & ns & ns & $\mathrm{ns}$ \\
\hline & Ratio $\mathrm{P} / \mathrm{I}$ of plateau & $0.30 \pm 0.041$ & $0.25 \pm 0.021$ & $0.28 \pm 0.060$ & $\$$ & ns & ns \\
\hline & Latency to plateau (s) & $0.95 \pm 0.168$ & $0.95 \pm 0.082$ & $1.35 \pm 0.583$ & ns & $\#$ & $\#$ \\
\hline & Duration of constriction (s) & $0.64 \pm 0.237$ & $0.59 \pm 0.145$ & $0.95 \pm 0.675$ & ns & ns & ns \\
\hline
\end{tabular}

[mean \pm standard deviation] for the 25 flashes experiment. $p_{1}$ - Comparison between healthy volunteers and diabetic subjects without CAN. $p_{2}$ - Comparison between healthy volunteers and diabetic subjects with CAN. $p_{3}$ - Comparison between diabetic subjects without and with CAN. ns - not significant, ${ }^{*}-p<0.05, \#-p<0.01$, and $\$-p<0.001$. 
There was no significant difference between the three groups with regard to age ( $p>$ $0.05)$. The duration of diabetes did not differ in the diabetic subjects with ( $20 \pm 15$ years) and without CAN (20 \pm 11 years), and HbA1c (\%) was not significantly different between these two groups $(10.2 \pm 2.1$ and $8.3 \pm 1.1)$.

Systolic blood pressure was significantly lower $(p<0.05)$ in healthy volunteers $(120 \pm$ $13 \mathrm{mmHg}$ ) when compared with diabetic subjects without CAN (143 $\pm 20 \mathrm{mmHg})$ and diabetic subjects with CAN (145 $\pm 14 \mathrm{mmHg})$. Moreover, diastolic blood pressure was significantly greater $(p<0.05)$ in diabetic subjects with CAN $(86 \pm 8 \mathrm{mmHg})$ than in diabetic subjects without CAN $(78 \pm 9 \mathrm{mmHg})$.

Table 2 shows the mean and SD values of the calculated parameter used to evaluate the response of the pupil reaction to 25 flashes and the statistical significance tests.

Pupil-iris ratio in the frame preceding the $10^{\text {th }}$ flash was significantly greater $(p<$ $0.001)$ in healthy volunteers $(0.33 \pm 0.050)$ than in diabetic subjects without CAN $(0.26 \pm$ $0.024)$, but no significant difference was found between the healthy volunteers and diabetic subjects with CAN.

The latency from the $10^{\text {th }}$ flash exposure to the start of constriction was not significantly different between the groups.

There was no difference between the groups in latency to the largest constriction of the pupil after the $10^{\text {th }}$ flash.

The pupil-iris ratio to the largest constriction was significantly greater $(p<0.05)$ in healthy volunteers $(0.28 \pm 0.049)$ than in diabetic subjects without CAN $(0.24 \pm 0.021)$, but no significant difference was found between the healthy volunteers and diabetic subjects with CAN.

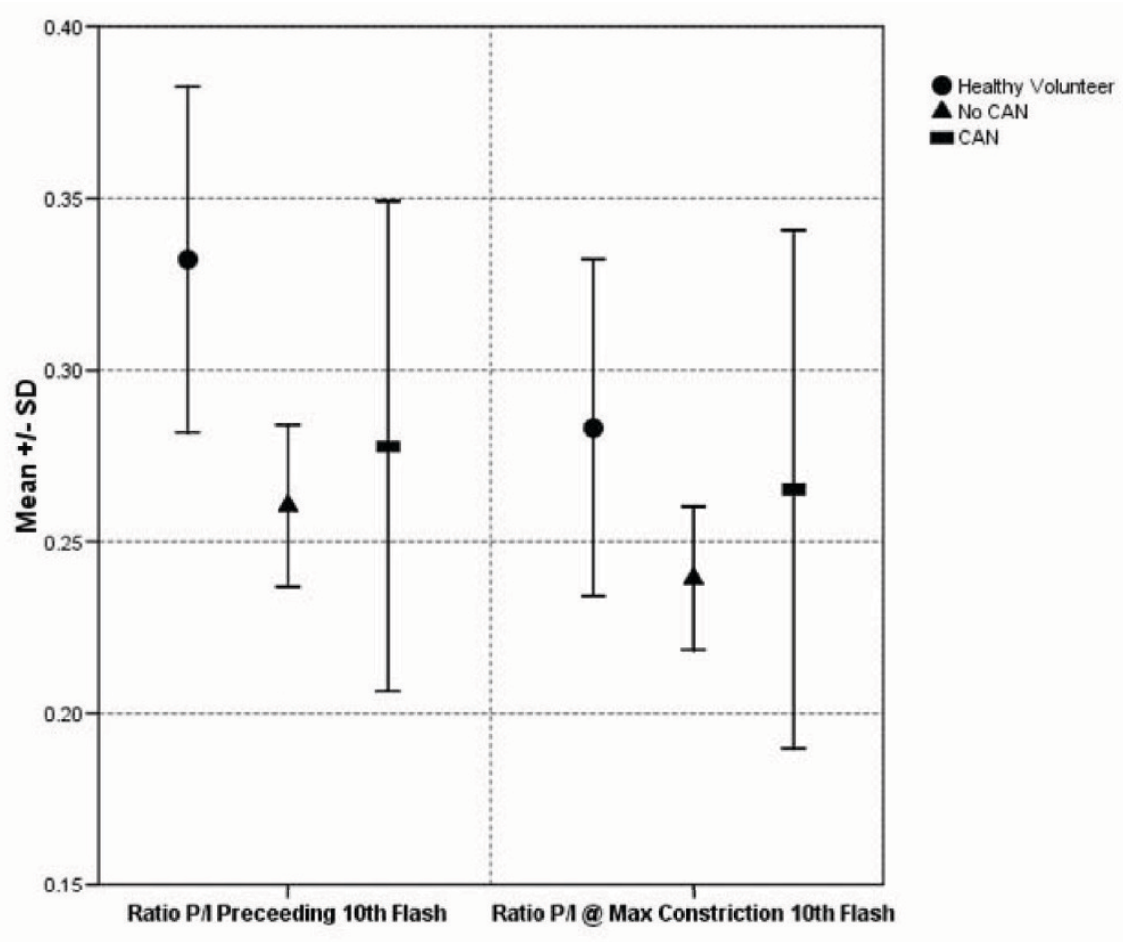

Figure 9 Comparison of mean and standard deviation values of the pupil-iris ratios (P/l) before the $10^{\text {th }}$ flash (\#3 in Figure 5) and at the largest constriction (\#4 in Figure 5). 
For the $10^{\text {th }}$ flash, no significant difference was found between diabetic groups.

The significant differences between groups, in pupil-iris ratios at $10^{\text {th }}$ flash for the second experiment, are illustrated in Figure 9.

The pupil-iris ratio one frame before the $25^{\text {th }}$ flash was also significantly greater $(p<$ $0.01)$ in healthy volunteers $(0.32 \pm 0.044)$ than in those diabetic subjects without CAN $(0.26 \pm 0.022)$, but no significant difference was found between the healthy volunteers and diabetic subjects with CAN.

There were no significant differences between the three groups in latency to constriction at the $25^{\text {th }}$ flash exposure, pupil-iris ratio, and latency to the largest constriction of the pupil after the $25^{\text {th }}$ flash.

During the recovery phase, the pupil-iris ratio at plateau was significantly greater $(p<$ $0.001)$ in healthy volunteers $(0.30 \pm 0.041)$ than in diabetic subjects without CAN $(0.28 \pm$ 0.060 ), but no significant difference was found between the healthy volunteers and diabetic subjects with CAN.

Moreover, the latency to plateau was significantly shorter $(p<0.01)$ in healthy volunteers $(0.95 \pm 0.168 \mathrm{~s})$ than in diabetic subjects with CAN $(1.35 \pm 0.583 \mathrm{~s})$.

Figure 10 shows a comparison of the mean and SD of the pupil-iris ratios at the $25^{\text {th }}$ flash among the three groups.

Duration of the constriction was not significantly different between the three groups.

For the $25^{\text {th }}$ flash, no significant difference was found between the diabetic groups, with an exception in the latency to plateau.

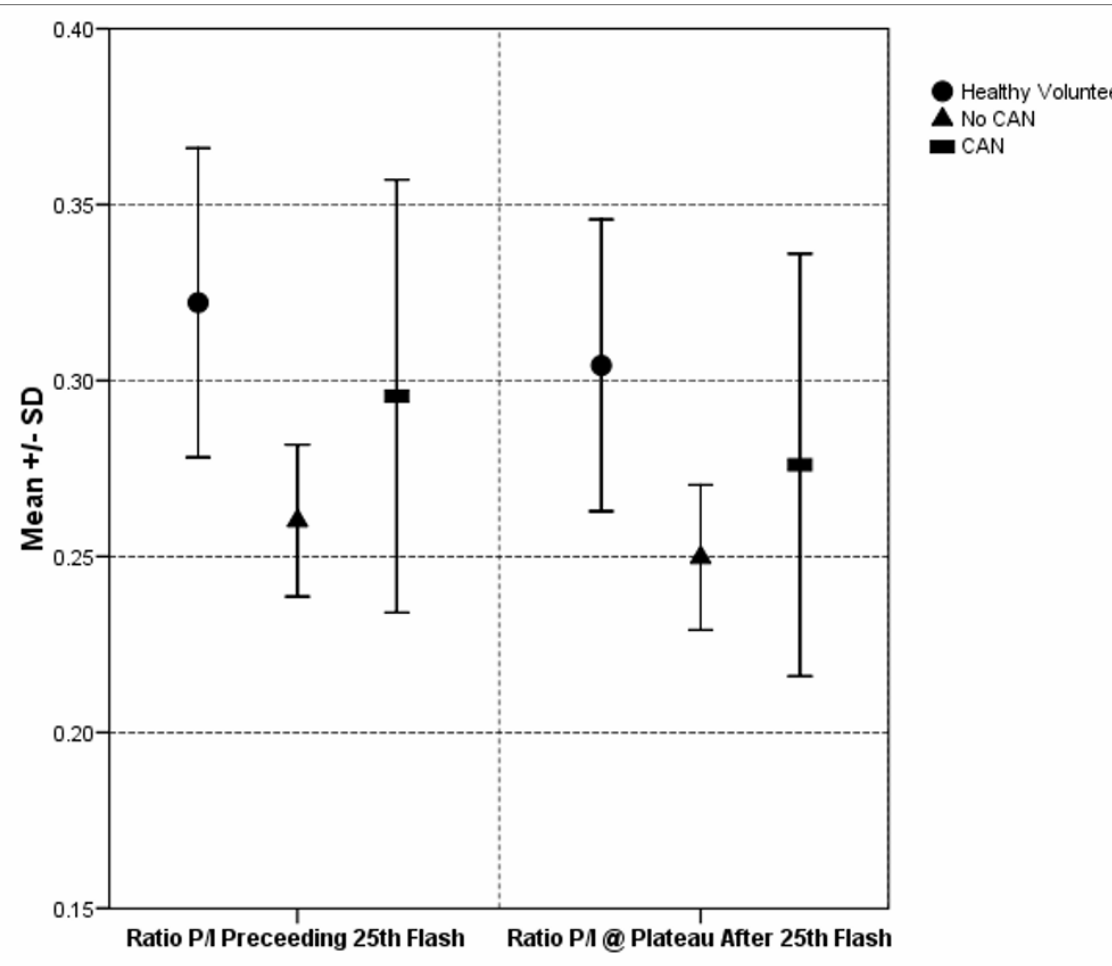

Figure 10 Comparison of mean pupil-iris ratios (P/I) preceding $25^{\text {th }}$ flash ( $\# 5$ in Figure 5 ) and during the plateau period (\#7 in Figure 5 ) in healthy volunteers (HV) and diabetic subjects with and without CAN (Error bars represent SD). 


\section{Discussion}

The results of the first experiment confirm the findings of previous studies [9,12-15] of a smaller resting pupil size and smaller reflex amplitude in subjects with diabetes.

According to Smith and Dewhirst [18], the resting pupil size is mainly under sympathetic control and radius reduction is a sign of diminished sympathetic outflow to the iris muscles. During the constriction phase, radius and time parameters mainly reflect parasympathetic function. However, both the systems are active during the recovery phase [15].

Smith and Smith [13] concluded that decreased reflex amplitudes in diabetic subjects cannot be ascribed to small pupil radius at rest, but are owing to a parasympathetic dysfunction. From our results in Table 1 comparing healthy volunteers and diabetic subjects (with and without CAN), a significant reduction in the pupil radius at baseline and amplitude of the pupil reflexes was found in both the diabetic groups. This might indicate that both sympathetic and parasympathetic dysfunction is affecting the pupillary reflex in these groups.

The significantly smaller initial pupil size seen in the diabetes group without CAN when compared with healthy subjects might be regarded as an early sign of involvement of the autonomic nerve system before cardiac manifestation of systemic autonomic neuropathy [9].

With regard to the second experiment, no comparison with previous findings was possible because no similar studies have been reported in the literature. The second experiment was found to lead to a gradual increase in the parasympathetic tone as a result of repeated light stimulation. This condition provides a different level of balance between parasympathetic and sympathetic nervous system, which is not linearly correlated to those of the first experiment. Although the two components of the autonomic nervous system may have abnormal tones, their combination may produce normal results during the first experiment. Moreover, the pupil muscles may perform well during a single flash administration, but this may not be true for repeated stimulation. The second experimental condition provided data on the flexibility of the pupil and autonomic nervous system.

Our results provide novel evidence of the increased prevalence of both parasympathetic and sympathetic autonomic dysfunction in diabetic subjects with and without $\mathrm{CAN}$, as evidenced by a significant reduction in the pupil-iris ratios and longer latency to start of constriction at the $10^{\text {th }}$ flash when compared with healthy volunteers. This shows that dynamic pupillometry may be a valuable tool for the early detection of these abnormalities. Failure to show a statistically significant difference between the diabetes groups in the second experiment may be accounted to the small number of subjects in the CAN group. There is a need for larger as well as prospective studies to better understand this relationship.

Dynamic pupillometry is a simple, inexpensive, and quick technique that requires minimal specialist training. It has the other added advantage over the battery of cardiovascular tests currently in use, in that it is not reliant on active subject participation and compliance. This study also suggests that it is a more sensitive measure of autonomic dysfunction than the conventional autonomic function tests. It also suggests that pupillary autonomic dysfunction occurs early, before a more generalized impairment of the autonomic nervous system, in diabetes. 
The pupil-iris ratio in darkness and the pupil reflex amplitude seem to be the dynamic pupillometry parameters most affected by alterations in the autonomic nervous system. Thus, they might be useful in detecting early autonomic dysfunction in high-risk groups.

\title{
Conclusions
}

In summary, dynamic pupillometry results indicate that this simple and non inexpensive approach can be used as an aiding tool during the clinical examination carried out in the general or specialized clinical settings. This method has the potential to significantly improve the outcome in detecting the early onset of autonomic dysfunction in subjects with diabetes mellitus.

\section{Competing interests}

The authors declare that they have no competing interests.

\section{Authors' contributions}

GLF, JLBM, HRG carried out the study, designed the study concept from a biomedical engineering perspective, designed the instrumentation, carried out the numerical investigations and image processing of pupil images, analyzed the data, and prepared the manuscript. RAG, SRH, ST designed the study concept from a clinical perspective, selected patients to take part in the study, supervised the work, and reviewed the manuscript. FKS designed the instrumentation and developed the algorithms for image processing. All authors have read and approved the final manuscript.

\author{
Acknowledgements \\ This work was supported by Paraná State Fundação Araucária and CAPES.
}

\section{Author Details}

'Electrical Engineering Department, Federal University of Paraná, Curitiba, PR - Brazil, 2School of Electrical Engineering and Applied Computer Sciences-CPGEI, Federal University of Technology-Paraná, Curitiba, PR - Brazil, 3Institute Biomedical Engineering, Electrical Engineering Department, Federal University Santa Catarina, Florianópolis, SC - Brazil and ${ }^{2}$ Royal Hallamshire Hospital, University of Sheffield, Sheffield, UK

Received: 5 August 2009 Accepted: 17 June 2010

Published: 17 June 2010

\section{References}

1. Vinik Al, Erbas T: Recognizing and treating diabetic autonomic neuropathy. Cleve Clin J Med 2001, 68:928-944.

2. Vinik Al, Ziegler D: Diabetic Cardiovascular Autonomic Neuropathy. Circulation 2007, 115:387-397.

3. American Diabetes Association and American Academy of Neurology: Report and recommendations of the San Antonio Conference on diabetic neuropathy (Consensus Statement). Diabetes 1988, 37:1000-1004.

4. Boulton AJ, Vinik Al, Arezzo JC, Bril V, et al:: Diabetic neuropathies: a statement by the American Diabetes Association. Diabetes Care 2005, 28(4):956-962.

5. Ewing DJ, Campbell IW, Clarke BF: The natural history of diabetic autonomic neuropathy. Q J Med 1980, 49:95-108.

6. Vinik Al, Maser RE, Mitchell BD, Freeman R: Diabetic Autonomic Neuropathy. Diabetes Care 2003, 5:1553-1579.

7. UKPDS - United Kingdom Prospective Diabetes Study Group: Association of glycaemia with macrovascular and microvascular complications of type 2 diabetes: prospective observational study. British Medical Journal 2000, 321:405-412.

8. Meeker M, Du R, Bacchetti P, Privitera CM, Larson MD, Holland MC, Manley G Pupil: Examination: Validity and Clinical Utility of an Automated Pupillometer. Journal of Neuroscience Nursing 2005, 37:34-40.

9. Pittasch D, Lobmann R, Behrens-Baumann W, Lehnert H: Pupil Signs of Sympathetic Autonomic Neuropathy in Patients with Type 1 Diabetes. Diabetes Care 2002, 25:1545-1550.

10. Fotiou F, Fountoulakis KN, Goulas A, Alexopoulos L, Palikaras A: Automated standardized pupillometry with optical method for purposes and clinical practice and research. Clinical Physiology 2000, 20:336-447.

11. Bremner FD, Smith SE: Pupil Abnormalities in Selected Autonomic Neuropathies. Journal of Neuro-Ophthalmolog 2006, 26:209-219.

12. Bremner $\mathrm{F}$, Smith $\mathrm{S}$ : Pupil findings in a consecutive series of 150 patients with generalized autonomic neuropathy. Journal Neurology, Neurosurgery and Psychiatry 2006, 77:1 163-1168.

13. Smith SA, Smith SE: Reduced pupillary light reflexes in diabetic autonomic neuropathy. Diabetologia 1983, 24:330-332.

14. Smith SA, Smith SE: Evidence for neuropatic aetiology in the small pupil of diabetes melittus. British Journal Ophthalmology 1983, 67:89-93.

15. Dütsch M, Marthol H, Michelson G, Neundöefer B, Hilz MJ: Pupillography refines diagnosis of diabetic autonomic neuropathy. Journal of the Neurological Sciences 2004, 222:75-81.

16. Cahill M, Eustace $P$, Jesus V: Pupillary autonomic denervation with increasing duration of diabetes melittus. British Journal Ophthalmology 2001, 85:1225-1230.

17. Eppens MC, Craig ME, Cusuamo J, Hings S, Chan AKF, Howard NJ, et al:: Prevalence of Diabetes Complications in Adolescents with Type 2 Compared with Type 1. Diabetes Care 2006, 29:1300-1306. 
18. Smith S, Dewhirst R: A simple diagnostic test for pupillary abnormality in diabetic autonomic neuropathy. Diabetic Medicine 1986, 3:38-41.

19. Ferrari GL, Marques JLB, Gandhi RA, Emery CJ, Tesfaye S, Heller SR, Schneider FK, Gamba HR: An Approach to the Assessment of Diabetic Neuropathy Based on Dynamic Pupillometry. In Proceedings of the 29th Annual International Conference of the IEEE Engineering in Medicine and Biology Society. 2007 Aug 23-26; Lion Lion: IEEE Engineering in Medicine and Biology Society; 2007:557-560.

20. Pfeifer W, Denker A: Pupillary examination: do I really need to look? Insight The Journal of the American Society of Ophthalmic Registered Nurses, Inc 2007, 32(2):13-18.

21. Gonzalez RC, Woods RE: Digital Image Processing 3rd edition. Massachusetts, Prentice Hall; 2008

22. O'Brien IA, O'Hare JP, Lewing Ig, Corrall RJ: The prevalence of autonomic neuropathy in insulin dependent diabetes: a controlled study based on heart rate variability. Q J Med 1986, 61:957-967.

doi: $10.1186 / 1475-925 X-9-26$

Cite this article as: Ferrari et al., Using dynamic pupillometry as a simple screening tool to detect autonomic neuropathy in patients with diabetes: a pilot study BioMedical Engineering OnLine 2010, 9:26

\section{Submit your next manuscript to BioMed Central} and take full advantage of:

- Convenient online submission

- Thorough peer review

- No space constraints or color figure charges

- Immediate publication on acceptance

- Inclusion in PubMed, CAS, Scopus and Google Scholar

- Research which is freely available for redistribution 